\title{
What did you choose just now? Chimpanzees' short-term retention of memories of their own behavior
}

Many recent comparative studies have addressed "episodic" memory in nonhuman animals, suggesting that birds, rodents, great apes, and others can remember their own behavior after at least a half-day delay. By contrast, despite numerous studies regarding long-term memory, few comparable studies have been conducted on short-term retention for own behavior. In the current study, we addressed the following question: Do chimpanzees remember what they have just done? Four chimpanzees performed matching-to-sample and visual search tasks on a routine basis and were occasionally (every four sessions) given a "recognition" test immediately after their response during visual search trials. Even though these test trials were given very rarely, all four chimpanzees chose the stimulus they selected in the visual search trials immediately before the test trial significantly more frequently than they chose the stimulus they selected in another distractor trial. Subsequent experiments ruled out the possibility that preferences for the specific stimuli accounted for the recognition test results. Thus, chimpanzees remembered their own behavior even within a short-term interval. This type of memory may involve the transfer of episodic information from working memory to longterm episodic-like memory (i.e., an episodic buffer). 
2 What did you choose just now?

3 Chimpanzees' short-term retention of memories of their own behavior

4 Masaki Tomonaga1) and Takaaki Kaneko2 $\square$

5 1) Primate Research Institute, Kyoto University, Kanrin 41-2, Inuyama, Aichi 484-8506, Japan

6 2) RIKEN Brain Science Institute, Wako, Saitama, Japan

7 Corresponding Author: Masaki Tomonaga 
Episodic memory is defined as long-term memory for personally experienced events (Tulving, 1985).

9 Some researchers have suggested that episodic memory should contain information about "what" occurred, 10 "when" it occurred, and "where" it occurred (e.g., Clayton \& Dickinson 1998; Skov-Rackette et al., 2006). Due to recent advances in comparative cognitive science, many species have been found to show some degree of this "episodic" memory (e.g., food-caching jays (Clayton \& Dickinson, 1998), rhesus macaques (Hampton et al., 2005; Hoffman et al., 2009), rats (Crystal \& Babb, 2008), and dolphins (Marcado III et al., 1998). For example, great apes showed "episodic" memory (Menzel, 1998; Schwartz et al., 2005; Martin-Ordas, 2010). Menzel (1998) reported that one symbol-trained chimpanzee remembered where an object was hidden in an outdoor enclosure for 16 hours.

However, controversy about whether these examples of long-term memory satisfy the criteria of human-like episodic memory persists (Suddendorf \& Busby, 2003; Tulving, 2005). Thus, the term "episodiclike" memory is often used to describe this type of memory in nonhuman animals (e.g., Clayton \& Dickinson, 1998). One reason for this is that many of the studies mentioned above seemingly lack components of "what," "when," and "where." For example, Hampton et al. (2005) administered a memory task to rhesus monkeys that was functionally equivalent to those used by Clayton and Dickinson (1998); however, unlike the latter's results with jays, Hampton et al. found intact memory for the "what" and "where" of past events but not for the 24 "when."

Most previous studies of episodic-like memory in nonhuman animals have focused intensively on the "long-term" retention of episodes, primarily because of the definition of episodic memory mentioned above. However, the other aspects of memory of past episodes should also be examined. One neglected area is the transition from working (or short-term) memory to long-term episodic-like memory. In terms of human working memory, Baddeley (2000) proposed the "episodic buffer," a link between the working and long-term memory systems. However, how the short-term retention of working memories is crystalized into long-term episodic-like memories in nonhuman animals remains unclear (cf. Zhou et al., 2012). To address this issue, research regarding the short-term properties of episodic-like memory is necessary.

Another aspect of episodic-like memory is the retroactive retrieval of incidentally encoded information. Humans can recall past events that have not been actively encoded, and several researchers have recently become interested in this aspect of memory in nonhuman animals, conducting relevant experimental studies (dogs: Fujita et al., 2012; rats: Zhou et al., 2012; pigeons: Singer \& Zentall, 2007; Zentall et al., 2001, 37 2008).

Zentall and his colleagues (Singer \& Zentall, 2007; Zentall et al., 2001, 2008) tested pigeons for episodic-like memory under the matching-to-sample paradigm. For example, pigeons were trained to match the left position to red and the right position to green (position-color matching) and to match yellow to vertical lines and blue to horizontal lines (color-line matching). After learning to perform these two types of matching tasks, they completed probe trials in which red and green choices were occasionally presented after participants

43 chose the vertical or horizontal stimulus in the color-line trials. When pigeons selected the left key for vertical

44 lines, they chose the red key and vice versa. Pigeons exhibited significantly better performance than would be 
45 expected by chance (71.6\%) during such probe trials (Singer \& Zentall, 2007). This result indicates that pigeons remember their own behavior even when they are unexpectedly prompted to do so. The same experimental paradigm was also used by Zhou et al. (2012), who also reported that rats can respond correctly to unexpected probe trials during maze-learning tasks. Some researchers have emphasized this unexpectedness in the episodic memory system (Zentall et al., 2008); however, pigeons underwent eight probe trials in each of 24 104-trial sessions in Singer and Zentall's study (2007). Given such a relatively large number of probe trials, pigeons might have learned the specific procedure for the probe trials or the probe trials might have become 52 less unexpected.

Consistent with the approach adopted by Zentall et al. (Singer \& Zentall, 2007; Zentall et al., 2001,

54 2008) and Zhou et al. (2012), we also focused on chimpanzees' retroactive retrieval of incidentally encoded information. To this end, we tested the short-term retention ability of chimpanzees for their own behavior. Chimpanzee participants performed matching-to-sample and visual search trials. In the matching trials, the chimpanzees were initially shown a single sample stimulus on a touch-screen monitor and were required to touch it. After a delay interval, two choice stimuli, one of which was the same as the sample, were presented. In contrast, the search display, which contained one target and five distractor stimuli, was presented during the visual search trials, and the chimpanzees were required to touch an odd item among the uniform distractors. As

61 a probe test, chimpanzees were occasionally presented with two stimuli after they selected the target in the visual search trial. One of the stimuli was the one that the subject had chosen from the search display, and the

63 other differed from the stimuli in the previous search display. These probe trials were presented once per test 64 session, and the test session appeared every four baseline sessions to maintain the rarity and unexpectedness of the probe trials. If the chimpanzees remembered their own choice just before the probe test, they could choose the "correct" stimulus during the probe trial.

\section{METHODS}

\section{Participants}

Four chimpanzees (Pan troglodytes) from the Primate Research Institute, Kyoto University (KUPRI), participated: Pendesa (female, 28 years old at the onset of the present experiment), Ayumu (male, 7 years old), Cleo (female, 7 years old), and Pal (female, 7 years old). As these chimpanzees have a long history of comparative cognitive experiments involving matching-to-sample and visual search tasks (Fagot \& Tomonaga, 1999; Goto et al., 2012; Tomonaga 2001; Tomonaga \& Imura, 2010; and Matsuzawa et al., 2006), it was not necessary to train them for these tasks before the present experiments. They live in a social group of 14 individuals in an environmentally enriched outdoor compound $\left(770 \mathrm{~m}^{2}\right)$ connected to the experimental room by a tunnel.

\section{Ethical Considerations}

The care and use of the chimpanzees adhered to the $2^{\text {nd }}$ edition of the Guide for the Care and Use of 
80 Welfare and Animal Care Committee of KUPRI and by the Animal Research Committee of Kyoto University

81 (Approval No. 07-1554). All procedures adhered to the Japanese Act on the Welfare and Management of

82 Animals.

\section{Apparatus and Stimuli}

Experimental sessions were conducted in a booth $(1.8 \times 2.15 \times 1.75 \mathrm{~m})$ in an experimental room. A 17-inch LCD monitor (IO-Data, Tokyo, Japan, Model LCD-AD171F-T, $1280 \times 1024$ pixels, pixel size: $0.264 \times$ $0.264 \mathrm{~mm}$ ) with a touch screen was placed on a wall of the booth (Fig. 1) at a viewing distance of approximately $40 \mathrm{~cm}$. A food reward was delivered via a food tray connected to a universal feeder located under the monitor (Bio-Medica Ltd., Osaka, Japan, Model BUF-310). Experimental events and data collection were controlled by a PC using a customized program written in Microsoft Visual Basic 6.0๑.

We prepared 2,500 color photographs $(6.0 \times 6.0 \mathrm{~cm}$ in size $)$ depicting various kinds of objects and scenes as stimuli. We did not control any of the parameters of these pictures, such as the distribution of colors, average brightness, and so on. These stimuli were presented only once to each chimpanzee.

\section{Procedure}

\section{Matching-to-sample trials}

Figure 2A shows the flow of a matching-to-sample trial. In each trial, a blue square $(3.0 \times 3.0 \mathrm{~cm})$ was presented at the bottom center of the monitor as a warning signal. When the chimpanzee touched the sample stimulus terminated it, and a green circle $(3.0 \mathrm{~cm}$ in diameter) was presented at a random location on the monitor. A single touch to this circle was followed by the presentation of the two choice stimuli. This interval was defined as a self-paced delay interval. Touching the choice stimulus that was the same as the sample delivered a food reward (a piece of apple or a raisin) followed by a chime. If the chimpanzee chose a stimulus that differed from the sample, this response was recorded as an error, and a buzzer sounded. The intertrial interval was $3 \mathrm{~s}$.

\section{Visual search trials}

As in the matching trials, each trial began with the presentation of a warning signal. After touching this, the search display was presented. We prepared two types of visual search trials. In the homogeneousdistractor trials, one target and five identical distractors were presented as a search display (Fig. 2B), whereas six different stimuli appeared in the heterogeneous-distractor trials (Fig. 2C). The chimpanzees were required to touch the target stimulus during the homogeneous-distractor trials, but any choice of stimulus was nondifferentially reinforced during the heterogeneous-distractor trials. When the chimpanzees touched the target

111 during the homogeneous-distractor trials and touched any stimulus during the heterogeneous-distractor trials,

112 the search display disappeared, and a green circle was presented at a random location, as in the matching trials.

113 Touching the green circle delivered a food reward accompanied by a chime. If the chimpanzees touched one of 
114 the distractors during the homogeneous trials (error response), the search display disappeared, and a buzzer

115 sounded. The presentation of the green circle was intended to equalize the flow of the trials across the 116 matching, visual search, and test trials (described later).

\section{7 "What-did-you-choose" test}

118 The third type of trial was called a "what-did-you-choose" test trial (Figs. 2D and 2E). In this trial,

119 two choice stimuli occasionally appeared after the self-paced delay interval following the visual search task.

120 One of the two stimuli was the one that the subject chose from the search display, and the other was the one that

121 was different from any stimulus in the previous search display. Any touch to a stimulus caused the termination

122 of the stimuli, but was not followed by feedback or a food reward. It should be noted that if the chimpanzees

123 made an error during the homogeneous-distractor visual search component of the test trials, the subsequent

124 two-choice component was cancelled.

125 Each baseline session consisted of 28 trials, in which the first four trials were matching trials. Among

126 the remaining 24 visual search trials, 16 were homogeneous- and eight were heterogeneous-distractor trials.

127 These trials were randomly presented. Test sessions were presented after every four sessions of the baseline

128 trials, and either a homogeneous or heterogeneous test trial appeared as $29^{\text {th }}$ trial. Each chimpanzee received 64

129 test sessions, i.e., one session per day, four or five times per week, yielding 64 test trials (32 for each type of

130 test trial). All stimulus positions were randomly assigned across trials. The correct stimulus position was also

131 randomized among trials.

\section{Control tests}

133 The present experiment used various kinds of stimuli, which were not controlled for color, brightness,

134 or content. Thus, some stimuli may have been more salient than others based on these low-level features. As is

135 the case for humans, chimpanzees' visual search patterns are controlled by salient "pop-out" features

136 (Tomonaga, 1993, 2001). To evaluate the effect of this phenomenon, we prepared two types of control tests. If

137 degree of salience controlled choices, one would hypothesize that (1) chimpanzees would choose the same

138 stimulus they had chosen previously when only the two choice stimuli presented during the "what-did-you-

139 choose" test trials were presented again and that (2) chimpanzees would choose the same stimulus they had

140 chosen previously when the visual search display that had appeared during the test trials was presented again. If

141 the chimpanzees' behavior were not governed simply by these non-memory stimulus factors, they would

142 choose randomly in the control test trials.

143 In the first type of control test trial, called the no-sample two-choice test, only two stimuli were

144 presented (Fig. 2F). These two stimuli were identical to those that appeared in the choice phase of the "what-

145 did-you-choose?" test trials (with both homogeneous- and heterogeneous-distractor visual search displays)

146 given to each chimpanzee. If the choice response in the test trial were controlled by the chimpanzees' stimulus

147 preference, they would choose the same stimulus selected during the test trials. In the other control test, we

148 presented the same search display that had been presented in the previous "what-did-you-choose" test trials 
149 with homogeneous and heterogeneous distractors, but in the form of a visual search trial, to determine whether

150 the chimpanzees chose the stimulus based on their own stimulus preferences under the heterogeneous-distractor

151 condition. If this were the case, the chimpanzees would choose the same stimulus when the same search display

152 was presented. We administered 32 additional test sessions to each chimpanzee. Each session consisted of two

153 matching trials, 20 visual search trials (12 with homogeneous distractors and eight with heterogeneous

154 distractors), two no-sample two-choice test trials, and two visual search test trials. Test trials were presented

155 randomly during a session, and no food reward or feedback was given after the choices in the test trials.

\section{RESULTS}

All data from each chimpanzee are presented in the supplementary file. All chimpanzees completed the experimental sessions (258 sessions on average, range: 255-260) within 578 days on average (range: 481664); test trials were given every 9 days. Participants performed very accurately on matching $(97.1 \%$, standard error of the mean, $S E M=0.7)$ and homogeneous visual search $(94.0 \%, S E M=1.3)$ trials. The self-paced delay interval lasted $1.05 \mathrm{sec}$, on average, across all types of trials. Figure 3 (black bars) shows the results of the test trials. All chimpanzees chose the stimulus that was chosen immediately before significantly more often than they chose the other stimulus under both distractor conditions (the significance level at 0.05 was $65.6 \%$, binomial test): $77.4 \%(S E M=1.5)$ for the homogeneous- and $85.2 \%(S E M=7.0)$ for the heterogeneousdistractor test trials, respectively.

Because the current experiments were conducted over a long period of time, it is possible that performance on the test trials improved during the course of the experimental sessions. To evaluate these learning effects, we divided the 32 test trials under each distractor condition into four eight-trial blocks and compared the performance across blocks. As shown in Table 1, no systematic improvement was observed across blocks. We found a significant difference in Cleo's performance under the homogeneous-distractor condition ( $p=0.038$, Fisher's exact probability test); however, when the data were analyzed based on the first 16 versus the second 16 trials $(68.8 \%$ vs. $81.25 \%)$, this difference was not significant $(p=0.685)$. $(98.4 \%, S E M=1.1)$ and visual search trials $(94.0 \%, S E M=1.7)$. For the first type of test trials (i.e., the nosample two-choice tests), the stimulus choice made by the chimpanzees was random in comparison with their previous choices $[41.4 \%(S E M=6.8)$ in the homogeneous-display control and $42.2 \%(S E M=2.7)$ in the

177 heterogeneous-display control test trials; see Fig. 3, gray bars]. Actually, the performance of two chimpanzees

178 (Ayumu and Pal) significantly differed from chance in the homogeneous-display control two-choice test trials, 179 but they avoided the stimulus that they had previously chosen. In the other control test of the repeated visual 180 search test trials (Table 2), the chimpanzees chose the same stimulus they had chosen in the previous 181 homogeneous-distractor test trial in $92.2 \%(S E M=2.7)$ of the trials. However, they chose the same stimulus as 182 in the previous heterogeneous-distractor test trial in only $21.1 \%(S E M=5.3)$ of the trials. Although one 183 chimpanzee exhibited significantly better performance (Ayumu, 31.3\%, $p<0.05$, binomial test) than would be expected by chance $(16.7 \%)$, the other three chimpanzees chose stimuli randomly. 
185

186

187

188

189

190

191

192

193

194

195

196

197

198

199

200

201

202

203

204

205

206

207

208

209

210

211

212

213

214

215

216

217

218

219

220

\section{DISCUSSION}

Irrespective of the type of distractors, all chimpanzees exhibited significantly better recognition of the stimulus chosen immediately before testing than would be expected by chance. These results indicate shortterm retention of memories of their own behavior. Furthermore, they performed at the level of chance on control test trials. These data suggest that the results of "what-did-you-choose" test trials cannot be explained by only the salience of the stimuli.

Our results clearly show that chimpanzees can remember choices they made immediately before an incidental memory task. Unlike the pigeons studied in previous research (Singer \& Zentall, 2007; Zentall et al., 2001, 2008), the chimpanzees in our study experienced a very long interval (one trial/9 days) between test trials. In contrast with the situation involving pigeons, this long inter-test interval may have made it less likely that the chimpanzees expected to be asked, "what did you choose?" immediately after an individual trial. Thus, the present results do not support rule learning or semantic memory (Singer \& Zentall, 2007). Their choice behavior during the test trials cannot be explained by simple association learning on the basis of differential reinforcement because the choice of the stimulus on the search display and the subsequent choice between the two stimuli were not actually differentially reinforced by a food or sound reward. Additionally, it would be difficult to explain our results in terms of non-memory strategies such as stimulus preference. The choices of the chimpanzees during the heterogeneous-distractor trials and the choice phase during the test trials were not consistent enough to explain our results. In the test trials, one of the two choice stimuli was selected from the stimuli in the previous search display. This may have led to different degrees of familiarity with the two choice stimuli, and the chimpanzees may have utilized these differences in the choice of a stimulus. This possibility should be examined further in the near future. Of course, it is still rather difficult to distinguish between semantic memory and episodic recollection using the current experimental procedure. It remains plausible that the chimpanzees chose the stimuli based on semantic memory ("that is the stimulus I chose") rather than based on episodic recollection ("I remember choosing that stimulus"). In the future, we should devise a new procedure to differentiate these possibilities more clearly.

Previous studies have reported that chimpanzees have the ability to form long-term episodic-like memories (e.g., Menzel, 1998). In our experiments, the delay interval between the choice behavior and the incidental question was only 1 second. Can our findings be connected with the previous results showing longterm retention of memories of their own behavior? Some researchers may argue for a critical gap between the short- and long-term properties of episodic memory, whereas others may not make this argument (Singer \& Zentall, 2007). We think that our results can be closely related to the "episodic buffer" in the components of working memory proposed by Baddeley (2000). This component is considered to act as a bridge between working memory and long-term episodic memory. Chimpanzees may temporally store memories of their own behavioral episodes in this episodic buffer. As Baddeley proposed, the episodic buffer plays a critical role in feeding information into and retrieving information from long-term episodic memory. Thus, we should focus on the temporal dynamics of episodic-like memory and its underlying mechanisms. However, these 
221 mechanisms remain unclear from the perspective of comparative cognition. More detailed comparative studies 222 should be conducted in the future.

223 In our experiment, only the "what" component of short-term episodic-like memory was examined, as 224 our experimental design did not allow us to determine the "when" and "where" components. For example, the 225 "when" component, was always fixed: the choice "just before" the current testing phase. Furthermore, the 226 "where" component could not be manipulated in our setting as we always tested the chimpanzees in the same 227 apparatus in the same room. Although controversy concerning the necessary conditions for testing episodic-like 228 memory in nonhuman animals persists (Zentall et al., 2008), we plan to modify the experimental procedure 229 enough to test the "when" and "where" components in a well-controlled setting. These further attempts will tell 230 us more about episodic-like memory in chimpanzees as well as about the evolution of episodic memory.

\section{CONCLUSION}

In the present study, we examined the ability of chimpanzees to recognize what they had just done 233 using matching-to-sample and visual search paradigms. In the memory-test trials, chimpanzees chose the 234 stimulus that they had chosen previously. These results seem to support the operation of short-term episodic-

235 like memory in chimpanzees. The comparative cognition domain has emphasized the long-term aspects of 236 episodic-like memory more than short-term storage of episodes, which may be attributable to the original 237 definition of episodic memory. However, the present results suggest the need for additional comparative 238 cognitive investigations of the temporal dynamics involved in role of episodic-like memory in the transition 239 from short-term to long-term storage. 


\section{ACKNOWLEDGMENTS}

241 We thank all of the staff of the Language and Intelligence Section and the Centre for Human Evolution

242 Modelling Research of the Primate Research Institute, Kyoto University for their comments, generous support, 243 and daily care of chimpanzees. 
244

245

246

247

248

249

250

251

252

253

254

255

256

257

258

259

260

261

262

263

264

265

266

267

268

269

270

271

272

273

274

275

276

277

278

279

280

\section{REFERENCES}

Baddeley A. 2000. The episodic buffer: A new component of working memory? Trends in Cognitive Science 4: 417-423

Clayton NS, Dickinson A. 1998. Episodic-like memory during cache recovery by scrub jays. Nature 395: 272274

Crystal JD, Babb SJ. 2008. Spatial memory in rats after 25 hours. Learning and Motivation 39: 278-284

Fagot J, Tomonaga M. 1999. Global-local processing in humans (Homo sapiens) and chimpanzees (Pan troglodytes): Use of a visual search task with compound stimuli. Journal of Comparative Psychology 113: $3-12$

Fujita K, Morisaki A, Takaoka A, Maeda T, Hori Y. 2012. Incidental memory in dogs (Canis familiaris): Adaptive behavioral solution in an unexpected memory test. Animal Cognition 15: 1055-1063

Goto K, Imura T, Tomonaga M. 2012. Perception of emergent configurations in humans (Homo sapiens) and chimpanzees (Pan troglodytes). Journal of Experimental Psychology: Animal Behavior Processes 38: $125-138$

Hampton RR, Hampstead BM, Murray EA. 2005. Rhesus monkeys (Macaca mulatta) demonstrate robust memory for what and where, but not when, in an open-field test of memory. Learning and Motivation 36: 245-259

Hoffman ML, Beran, MJ, Washburn DA. 2009. Memory for "what," "where" and "when" information in rhesus monkeys (Macaca mulatta). Journal of Experimental Psychology: Animal Behavior Processes 35: $143-152$

Martin-Ordas G, Haun D, Colmenares F Call J. 2010. Keeping track of time: Evidence of episodic-like memory in great apes. Animal Cognition 13: 331-340

Matsuzawa T, Tomonaga M, Tanaka M, eds. 2006. Cognitive development in chimpanzees. Springer, Tokyo

Menzel CR. 1999. Unprompted recall and reporting of hidden objects by a chimpanzee (Pan trogolodytes). Journal of Comparative Psychology 113: 426-43

Mercado III E, Murray SO, Uyeyama RK, Pack AA, Herman LM. 1998. Memory for recent actions in the bottlenosed dolphin (Tursiops truncates): Repetition of arbitrary behaviors using the abstract rule. Animal Learning and Behavior 26: 210-218

Schwartz BL, Hoffman ML, Evans S. 2005. Episodic-like memory in a gorilla: A review and new findings. Learning and Motivation 36: 226-244

Singer RA, Zentall TR. 2007. Pigeons learn to answer the question "where did you just peck?" and can report peck location when unexpectedly asked. Learning and Behavior 35: 184-189

Skov-Rackette SI, Miller NY, Shettleworth SJ. 2006. What-where-when memory in pigeons. Journal of Experimental Psychology: Animal Behavior Processes 32: 345-358

Suddendorf T, Busby J. 2003. Mental time travel in animals? Trends in Cognitive Sciences, 9: 391-396

Tomonaga M. 1993. A search for search asymmetry in chimpanzees (Pan troglodytes). Perceptual and Motor Skills 76: 1287-1295 
281 Tomonaga M. 2001. Investigating visual perception and cognition in chimpanzees (Pan troglodytes) through

282

283

284

285

286

287

288

289

290

291

292

293

294

295

296 visual search and related tasks: From basic to complex processes. In: Matsuzawa T, ed. Primate origin of human cognition and behavior. Springer, Tokyo, pp 55-86

Tomonaga M, Imura T. 2010. Pacman in the sky with shadows: The effect of cast shadows on the perceptual completion of occluded figures by chimpanzees and humans. Behavioral and Brain Function 6: 38. doi:10.1186/1744-9081-6-38.

Tulving E. 1985. How many memory systems are there? American Psychologist 40: 385-398

Tulving E. 2005. Episodic memory and autonoesis: Uniquely human? In: Terrace HS \& Metcalfe J, eds. The missing link in cognition: Origins of self-reflective consciousness. Oxford University Press, Oxford, pp. 3-56

Zentall TR, Clement TS, Bhatt RS, Allen J. 2001. Episodic-like memory in pigeons. Psychonomic Bulletin and Review 8: 685-690

Zentall TR, Singer RA, Stagner JP. 2008. Episodic-like memory: Pigeons can report location pecked when unexpectedly asked. Behavioral Process 79: 93-98

Zhou W, Hohmann AG, Crystal JD. 2012. Rats answer an unexpected question after incidental encoding. Current Biology, 22: 1149-1153 
297 Table S1. Raw data for each chimpanzee in the present experiment. Microsoft Excel 2003 file format (.xls) 
298 Figure Captions

299 Figure 1. Chimpanzee Cleo performing the "what-did-you-choose?" test. Photo Courtesy: Masaki Tomonaga 300 (Kyoto University)

301 Figure 2. Flow of each type of trial in the present experiment. Photo Courtesy: Masaki Tomonaga (Kyoto 302 University)

303 Figure 3. Mean percentage of correct choices during the "what-did-you-choose?" tests and the no-sample 304 control tests. Error bars indicate the standard errors. Data from each chimpanzee are also shown. 


\section{Table $\mathbf{1}$ (on next page)}

Learning effects in the "what-did-you-choose" test trials.

Each number represents the number of correct trials in each eight-trial block. 


\section{PeerJ Reviewing Manuscript}

Table 1. Learning effects in the "what-did-you-choose" test trials. Each number represents the number of correct trials in each eight-trial block.

\begin{tabular}{|c|c|c|c|c|c|c|c|c|}
\hline \multirow[b]{2}{*}{$\begin{array}{l}\text { Eight- } \\
\text { trial } \\
\text { block }\end{array}$} & \multicolumn{2}{|c|}{ Pendesa } & \multicolumn{2}{|c|}{ Aуumu } & \multicolumn{2}{|c|}{ Cleo } & \multicolumn{2}{|c|}{$\mathrm{Pal}$} \\
\hline & $\begin{array}{l}\text { homo } \\
\text { DSTs }\end{array}$ & $\begin{array}{l}\text { hetero } \\
\text { DSTs }\end{array}$ & $\begin{array}{l}\text { homo } \\
\text { DSTs }\end{array}$ & $\begin{array}{l}\text { hetero } \\
\text { DSTs }\end{array}$ & $\begin{array}{l}\text { homo } \\
\text { DSTs }\end{array}$ & $\begin{array}{l}\text { hetero } \\
\text { DSTs }\end{array}$ & $\begin{array}{l}\text { homo } \\
\text { DSTs }\end{array}$ & $\begin{array}{l}\text { hetero } \\
\text { DSTs }\end{array}$ \\
\hline 1 & 5 & 5 & 8 & 7 & 3 & 8 & 8 & 8 \\
\hline 2 & 6 & 6 & 6 & 7 & 8 & 8 & 5 & 8 \\
\hline 3 & 7 & 6 & 4 & 5 & 7 & 7 & 5 & 7 \\
\hline 4 & 6 & 5 & 7 & 6 & 6 & 8 & 8 & 8 \\
\hline $\begin{array}{l}\text { Fisher's } \\
\text { Exact } p\end{array}$ & 0.942 & 1.000 & 0.145 & 0.791 & 0.038 & 1.000 & 0.053 & 1.000 \\
\hline
\end{tabular}

homoDSTs: trials with homogeneous distractors; heteroDSTs: trials with heterogeneous distractors 
Table 2 (on next page)

Results of the second control test sessions.

The percentages of correct trials under baseline conditions and the percentage of trials in which chimpanzees chose the same stimuli they had chosen previously in the repeated visual search test trials. 


\section{PeerJ Reviewing Manuscript}

Table 2. Results of the second control test sessions. The percentages of correct trials under baseline conditions and the percentage of trials in which chimpanzees chose the same stimuli they had chosen previously in the repeated visual search test trials.

\begin{tabular}{lccrr}
\hline & \multicolumn{2}{c}{ Baseline } & \multicolumn{2}{c}{ Test } \\
\hline Chimpanzee & MTS & \multicolumn{2}{l}{$\begin{array}{l}\text { Visual Search } \\
\text { (homoDSTs) }\end{array}$} & \multicolumn{2}{c}{ Visual Search } \\
homoDSTs & heteroDSTs \\
\hline Pendesa & 98.4 & 94.5 & 96.9 & 6.3 \\
Ayumu & 100 & 98.4 & 96.9 & 31.3 \\
Cleo & 95.3 & 90.4 & 87.5 & 21.9 \\
Pal & 100 & 92.7 & 87.5 & 25 \\
\hline Average & 98.4 & 94 & 92.2 & 21.1 \\
SE & 1.1 & 1.7 & 2.7 & 5.3 \\
\hline
\end{tabular}

homoDSTs: trials with homogeneous distractors; heteroDSTs: trials with heterogeneous distractors 


\section{Figure 1}

Chimpanzee Cleo performing the "what-did-you-choose?" test.

Photo Courtesy: Masaki Tomonaga (Kyoto University)

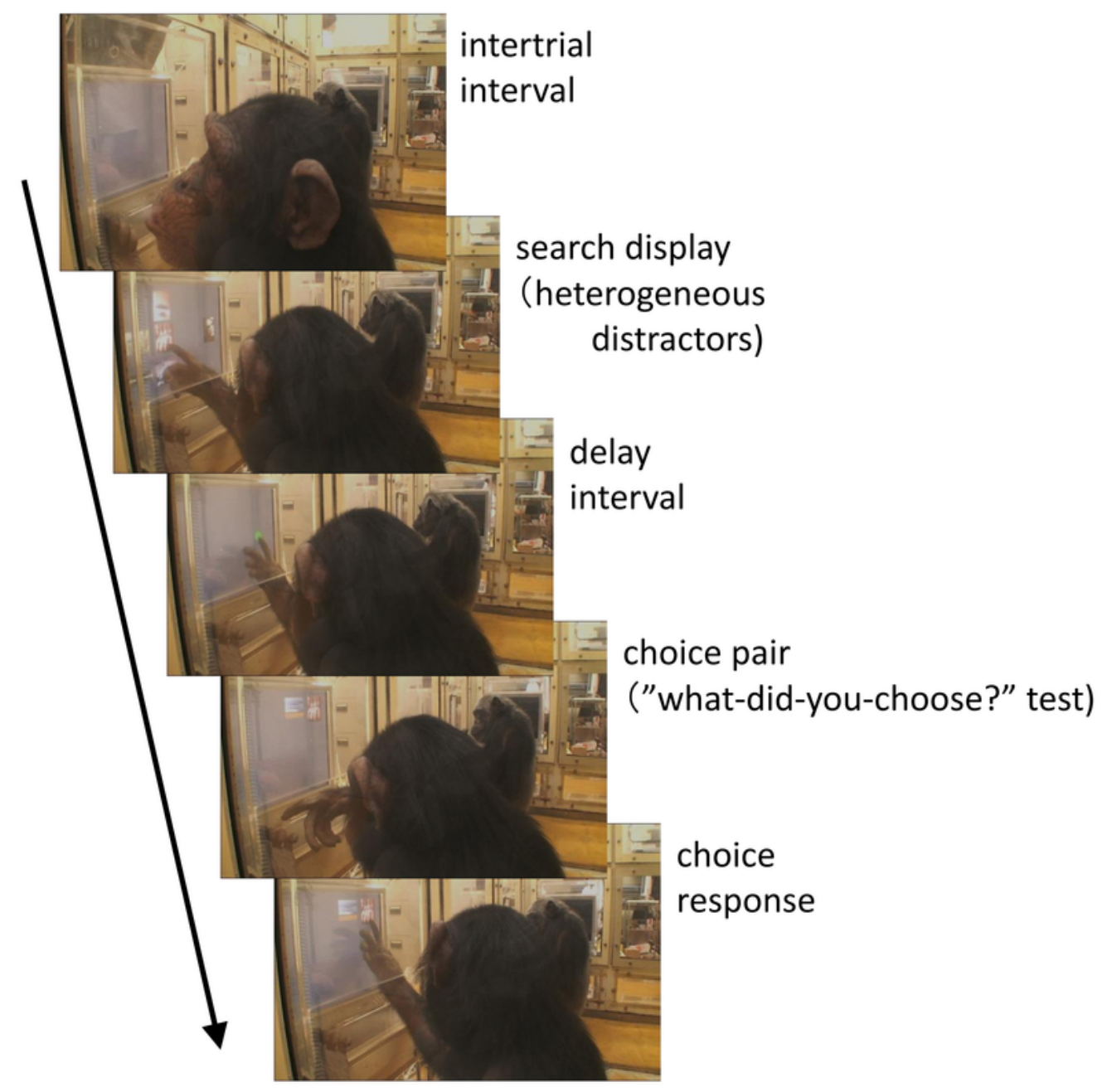


Figure 2 (on next page)

Flow of each type of trial in the present experiment.

Photo Courtesy: Masaki Tomonaga (Kyoto University) 
matching trial

(A)
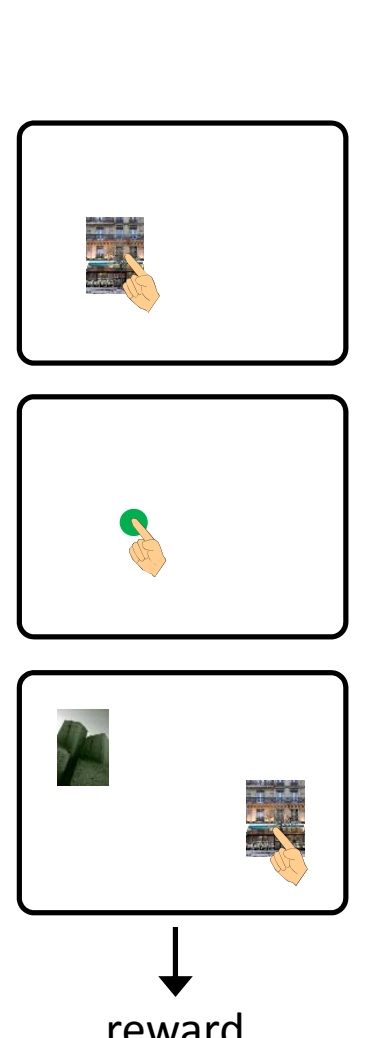

visual search trial

(B)
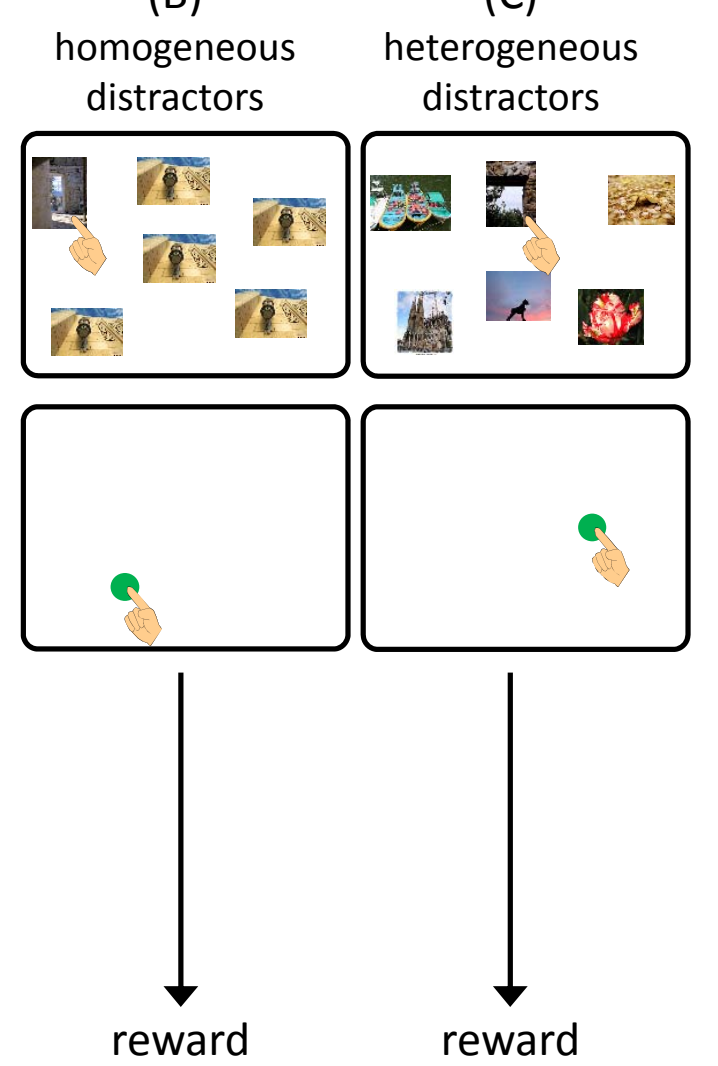

"what-did-you-choose?" test trial

(E)

homogeneous heterogeneous

distractors distractors
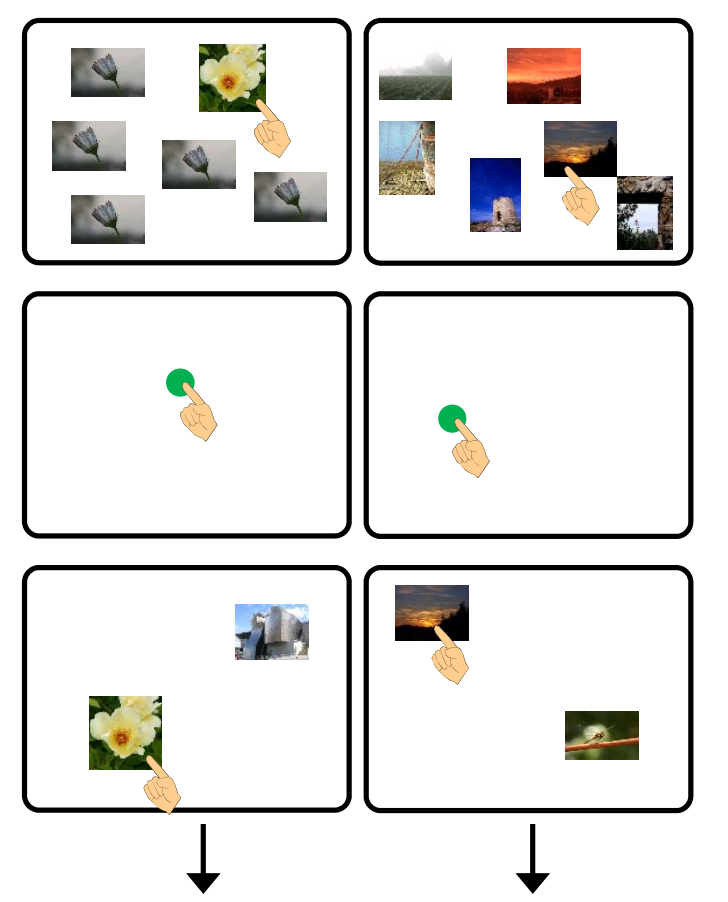

no feedback no-sample two-choice test

(F)
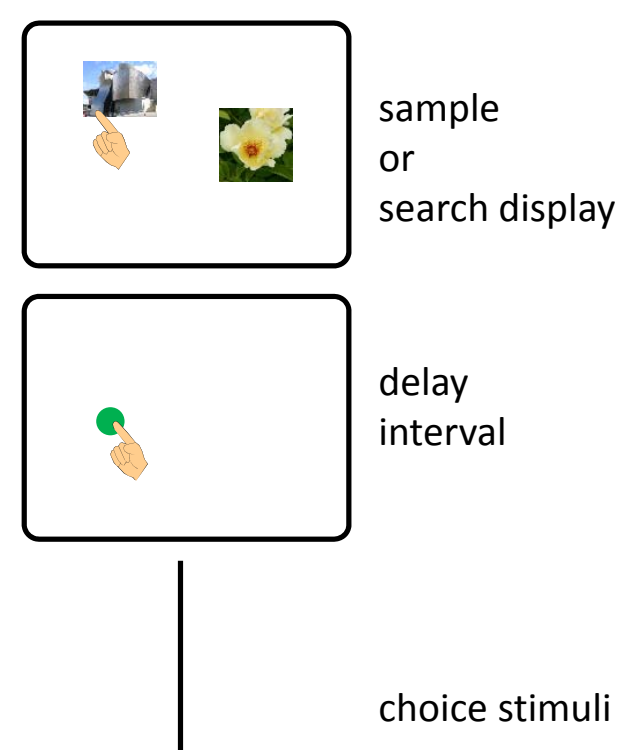

choice stimuli 
Figure 3 (on next page)

Mean percentage of correct choices during the "what-did-you-choose?" tests and the no-sample control tests.

Error bars indicate the standard errors. Data from each chimpanzee are also shown. 


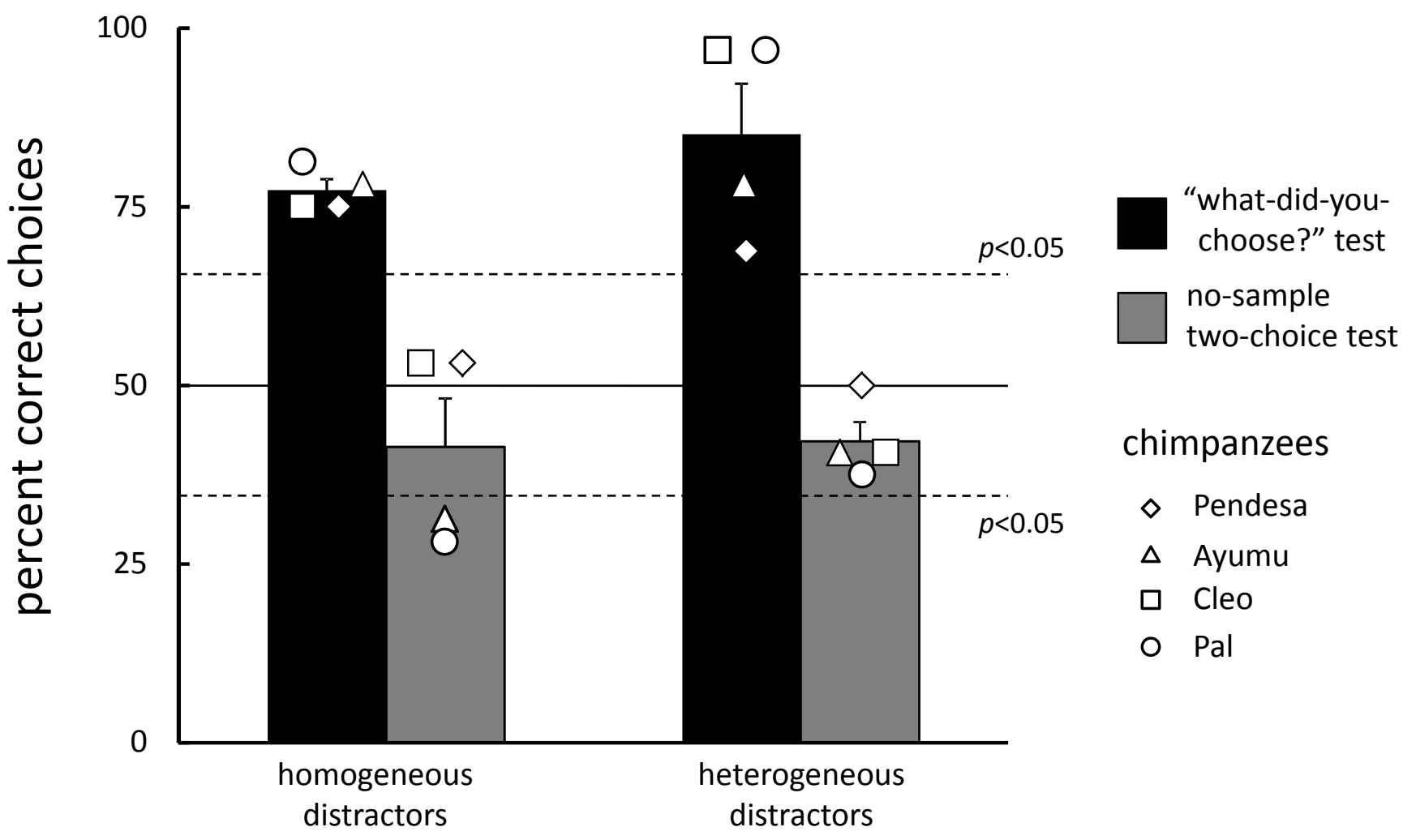

